WoJCIECH M. ZAJĄCZKOWski (Warszawa)

\title{
SOLVABILITY OF THE POISSON EQUATION IN WEIGHTED SOBOLEV SPACES
}

Abstract. The aim of this paper is to prove the existence of solutions to the Poisson equation in weighted Sobolev spaces, where the weight is the distance to some distinguished axis, raised to a negative power. Therefore we are looking for solutions which vanish sufficiently fast near the axis. Such a result is useful in the proof of the existence of global regular solutions to the Navier-Stokes equations which are close to axially symmetric solutions.

1. Introduction. The aim of this paper is to prove the existence of solutions to the Poisson equation in weighted Sobolev spaces $H_{-\mu}^{l}(\Omega), l \in$ $\mathbb{N}_{0} \equiv \mathbb{N} \cup\{0\}, \mu \in \mathbb{R}_{+}$, where $\Omega \subset \mathbb{R}^{3}$ is a bounded domain which contains a (segment of a) distinguished axis $L$. We assume that the weight is a negative power of the distance to $L$.

We consider the Dirichlet problem

$$
\begin{array}{ll}
-\Delta u=f & \text { in } \Omega, \\
u=0 & \text { on } S=\partial \Omega .
\end{array}
$$

We assume that $L$ meets $S$ at two points: $s_{1}$ and $s_{2}$.

Definition 1.1. By $H_{-\mu}^{l}(\Omega), l \in \mathbb{N}_{0}, \mu \in \mathbb{R}_{+}$, we denote the closure of the set of $C^{\infty}(\Omega)$-functions vanishing in a neighbourhood of $L$ in the norm

$$
\|u\|_{H_{-\mu}^{l}(\Omega)}=\left(\sum_{|\alpha| \leq l} \int_{\Omega}\left|D_{x}^{\alpha} u(x)\right|^{2} \varrho(x)^{2(-\mu+|\alpha|-l)} d x\right)^{1 / 2},
$$

where $|\alpha|=\alpha_{1}+\alpha_{2}+\alpha_{3}, D_{x}^{\alpha}=\partial_{x_{1}}^{\alpha_{1}} \partial_{x_{2}}^{\alpha_{2}} \partial_{x_{3}}^{\alpha_{3}}, \alpha_{i} \in \mathbb{N}_{0}, i=1,2,3, \varrho(x)=$ $\operatorname{dist}(x, L)$.

The main result of this paper is the following

2010 Mathematics Subject Classification: 35K05, 35K20.

Key words and phrases: Poisson equation, weighted Sobolev spaces, existence in weighted Sobolev spaces. 
Theorem 1.2. Assume that $f \in H_{-\mu}^{l}(\Omega), l \in \mathbb{N}_{0}, \mu \in \mathbb{R}_{+} \backslash \mathbb{Z}$. Assume that $S \in C^{l+2}$. Then there exists a solution to problem (1.1) such that $u \in$ $H_{-\mu}^{l+2}(\Omega)$ and the estimate

$$
\|u\|_{H_{-\mu}^{l+2}(\Omega)} \leq c\|f\|_{H_{-\mu}^{l}(\Omega)} .
$$

holds, where $c$ does not depend on $u$ and $f$.

In this paper we prove the existence only because estimate (1.2) is already known (see [2, 7]). The well known method to solve problem (1.1) is to show the existence of weak solutions to problem (1.1) defined by the integral identity

$$
\int_{\Omega} \nabla u \cdot \nabla \varphi d x=\int_{\Omega} f \varphi d x
$$

which holds for any smooth function vanishing on $S$.

Assuming that $f \in L_{2}(\Omega)$ and applying the Galerkin method it is well known that we have the existence of weak solutions to (1.1) belonging to $H^{1}(\Omega)$ and satisfying the estimate

$$
\|u\|_{H^{1}(\Omega)} \leq c\|f\|_{L_{2}(\Omega)},
$$

where $c$ does not depend on $u$ and $f$.

Similarly, assuming $f \in H^{l}(\Omega), S \in C^{l+2}, l \in \mathbb{N}_{0}$ and applying the regularization technique (see [3, 4]) we can prove the existence of solutions to $(1.1)$ in $H^{l+2}(\Omega)$ and the estimate

$$
\|u\|_{H^{l+2}(\Omega)} \leq c\|f\|_{H^{l}(\Omega)},
$$

where $c$ does not depend on $u$ and $f$.

The above procedure is possible because $H^{l+2}(\Omega) \subset H^{1}(\Omega)$, and the technique of increasing regularity is well developed. In our case we also have $H_{-\mu}^{l+2}(\Omega) \subset H^{1}(\Omega)$ but elements of $H_{-\mu}^{l+2}(\Omega)$ must satisfy some structural restrictions which are expressed by an appropriate vanishing of solutions to (1.1) near the axis $L$. Hence to prove the existence of solutions to (1.1) in $H_{-\mu}^{l+2}(\Omega)$ we have to find a way of selecting elements from $H^{1}(\Omega)$ satisfying the structural conditions.

We may use two approaches to prove Theorem 1.2. The first is direct by employing the methods and ideas from [1, 5, 12]. The second, which is used in this paper, can be split into a few steps. First we show the existence of solutions to (1.1) in $H^{l+2}$ in a subdomain of $\Omega$ located at a positive distance from $L$. Next we appropriately extend the solution to a neighbourhood of $L$ in such a way that the extended solution belongs to $H_{-\mu}^{l+2}(\Omega)$.

Then using estimate (1.2) and applying an appropriate density argument we prove Theorem 1.2. This idea will be described more precisely in Section 2 . 
We should also mention that the main tool in the proof of Theorem 1.2 is the technique of regularizers (see [4]). This is connected with the fact that the crucial estimate (1.2) in weighted spaces is only obtained locally.

To prove Theorem 1.2 we need

Lemma 1.3. Assume that $f \in H^{l}(\Omega), S \in C^{l+2}, l \in \mathbb{N}_{0}$. Then there exists a solution to problem (1.1) such that $u \in H^{l+2}(\Omega)$ and

$$
\|u\|_{H^{l+2}(\Omega)} \leq c\|f\|_{H^{l}(\Omega)},
$$

where $c$ does not depend on $u$ and $f$.

The techniques and methods used in this paper are important for examining nonstationary equations in similar weighted Sobolev spaces (see [8]).

In a series of papers (see [6, 9, 10, 11]) we proved the existence of global regular solutions to the Navier-Stokes equations which are close to axially symmetric solutions. To show those results we need the weighted Sobolev spaces introduced in this paper, because weights with negative powers appear naturally in estimates necessary for the existence of solutions.

2. Existence in $\mathbb{R}^{3}$. First we shall restrict our considerations to the case $l=0$. Let $A>0$ be given. Consider the cylinder

$$
C_{A}=\left\{x \in \mathbb{R}^{3}:\left|x^{\prime}\right|<A, x_{3} \in \mathbb{R}\right\},
$$

where $x^{\prime}=\left(x_{1}, x_{2}\right),\left|x^{\prime}\right|=\sqrt{x_{1}^{2}+x_{2}^{2}}$, and $x=\left(x_{1}, x_{2}, x_{3}\right)$ is the Cartesian system in $\mathbb{R}^{3}$.

Let $R>0$ be given. Then we consider the problem

$$
\begin{array}{ll}
-\Delta u=f & \text { in } C_{R}, \\
u=0 & \text { on } \partial C_{R}, \\
u \rightarrow 0 & \text { as }\left|x_{3}\right| \rightarrow \infty,
\end{array}
$$

where $\partial C_{R}=\left\{x \in \mathbb{R}^{3}:\left|x^{\prime}\right|=R, x_{3} \in \mathbb{R}\right\}$.

To prove the existence of solutions to problem (2.1) in weighted Sobolev spaces we define an approximate solution. Let $0<\delta<R$ be given. Let $C_{R, \delta}=C_{R} \backslash \bar{C}_{\delta}$, where $\bar{C}_{\delta}$ is the closure of $C_{\delta}$. Then we consider the problem

$$
\begin{array}{ll}
-\Delta u_{\delta}=f_{\delta} & \text { in } C_{R, \delta}, \\
u_{\delta}=0 & \text { on } \partial C_{R, \delta}, \\
u_{\delta} \rightarrow 0 & \text { as }\left|x_{3}\right| \rightarrow \infty .
\end{array}
$$

We have

Lemma 2.1. Assume that $f_{\delta} \in L_{2}\left(C_{R, \delta}\right)$. Then there exists a unique solution to (2.2) such that $u_{\delta} \in H^{2}\left(C_{R, \delta}\right)$ and

$$
\left\|u_{\delta}\right\|_{H^{2}\left(C_{R, \delta}\right)} \leq c\left\|f_{\delta}\right\|_{L_{2}\left(C_{R, \delta}\right)},
$$

where $c$ does not depend on $u_{\delta}$ and $f_{\delta}$. 
Let us introduce the cylindrical coordinates $(r, \varphi, z)$ by the relations $x_{1}=$ $r \cos \varphi, x_{2}=r \sin \varphi, x_{3}=z$.

Since we are looking for solutions to the Poisson equation in $\mathbb{R}^{3}$ but we are not interested in their behaviour as $\left|x^{\prime}\right| \rightarrow \infty$ we extend $u_{\delta}$ by zero for $r>R$. For this purpose we introduce a smooth function $\zeta_{R}\left(x^{\prime}\right)$ such that $\zeta_{R}\left(x^{\prime}\right)=1$ for $\left|x^{\prime}\right| \leq \frac{3}{4} R$ and $\zeta_{R}\left(x^{\prime}\right)=0$ for $\left|x^{\prime}\right| \geq R$. Let $u_{\delta}^{\prime}=u_{\delta} \zeta_{R}$, $f_{\delta}^{\prime}=f_{\delta} \zeta_{R}$. Then problem (2.2) takes the form

$$
\begin{array}{ll}
-\Delta u_{\delta}^{\prime}=f_{\delta}^{\prime}-2 \zeta_{R, x^{\prime}} u_{\delta, x^{\prime}}-\zeta_{R, x^{\prime} x^{\prime}} u_{\delta} \equiv f_{\delta}^{\prime \prime} & \text { in } \mathbb{R}^{3} \backslash \bar{C}_{\delta}, \\
u_{\delta}^{\prime}=0 & \text { on } \partial C_{\delta}, \\
u_{\delta}^{\prime}=0 & \text { for } r>R, \\
u_{\delta}^{\prime} \rightarrow 0 & \text { as }\left|x_{3}\right| \rightarrow \infty .
\end{array}
$$

In view of Lemma 2.1 we have $f_{\delta}^{\prime \prime} \in L_{2}\left(\mathbb{R}^{3} \backslash \bar{C}_{\delta}\right)$ and there exists a solution to problem (2.4) such that $u_{\delta}^{\prime} \in H^{2}\left(\mathbb{R}^{3} \backslash \bar{C}_{\delta}\right)$ and

$$
\left\|u_{\delta}^{\prime}\right\|_{H^{2}\left(\mathbb{R}^{3} \backslash \bar{C}_{\delta}\right)} \leq c\left\|f_{\delta}^{\prime}\right\|_{L_{2}\left(\mathbb{R}^{3} \backslash \bar{C}_{\delta}\right)} .
$$

To prove the existence of solutions to problem (1.1) in the weighted spaces $H_{-\mu}^{l}$ introduced in Definition 1.1 we extend $f_{\delta}^{\prime \prime}$ and $u_{\delta}^{\prime}$ by zero for $r<\delta$. Let us denote the extended functions by $\bar{f}_{\delta}$ and $\bar{u}_{\delta}$. Additionally we assume that $\left.f_{\delta}^{\prime}\right|_{r=\delta}=0$. Then $(2.4)_{1}$ implies that $\left.\Delta u_{\delta}^{\prime}\right|_{r=\delta}=0$. To show that $u_{\delta}^{\prime} \in$ $H_{-\mu}^{2}\left(\mathbb{R}^{3}\right)$ we assume that $\delta<\frac{3}{4} R$, and we define

$$
\begin{array}{ll}
v_{\delta}=\left.u_{\delta}^{\prime}\right|_{x=x(r, \varphi, z)}, & h_{\delta}=\left.f_{\delta}^{\prime \prime}\right|_{x=x(r, \varphi, z)}, \\
\bar{v}_{\delta}=\left.\bar{u}_{\delta}\right|_{x=x(r, \varphi, z)}, & \bar{h}_{\delta}=\left.\bar{f}_{\delta}\right|_{x=x(r, \varphi, z)} .
\end{array}
$$

Then problem (2.4) takes the form

$$
\begin{aligned}
& -\left(\frac{1}{r}\left(r \bar{v}_{\delta, r}\right)_{, r}+\frac{1}{r^{2}} \bar{v}_{\delta, \varphi \varphi}+\bar{v}_{\delta, z z}\right)=\bar{h}_{\delta} \quad \text { in } \mathbb{R}^{3}, \\
& \left.\bar{v}_{\delta}\right|_{r \leq \delta}=0, \\
& \left.\bar{h}_{\delta}\right|_{r \leq \delta}=0, \\
& \bar{v}_{\delta} \rightarrow 0 \quad \text { as }|z| \rightarrow \infty, r>R .
\end{aligned}
$$

From $(2.6)_{2}$ it follows that $\bar{v}_{\delta, \varphi \varphi}=0$ and $\bar{v}_{\delta, z z}=0$ for $r \leq \delta$. Moreover, in view of Lemma 2.1 we have $(1 / r)\left(r \bar{v}_{\delta, r}\right)_{, r} \in L_{2}\left(\mathbb{R}^{3}\right)$, and $(2.6)_{1}$ implies

$$
\frac{1}{r}\left(r \bar{v}_{\delta, r}\right)_{, r}=0 \quad \text { for } r \leq \delta .
$$

Let $\mu \in \mathbb{R}_{+}$. Then $\bar{f}_{\delta} \in L_{2,-\mu}\left(\mathbb{R}^{3}\right)$. To apply [7] we should know that $\bar{v}_{\delta} \in$ $H_{-\mu}^{2}\left(\mathbb{R}^{3}\right)$. From the properties of $\bar{v}_{\delta}$ we have $\bar{v}_{\delta, \varphi \varphi} \in L_{2,-\mu}\left(\mathbb{R}^{3}\right), \bar{v}_{\delta, z z} \in$ $L_{2,-\mu}\left(\mathbb{R}^{3}\right)$ and $(1 / r)\left(r \bar{v}_{\delta, r}\right)_{, r} \in L_{2,-\mu}\left(\mathbb{R}^{3}\right)$. Moreover,

$$
\left\|\frac{1}{r}\left(r \bar{v}_{\delta, r}\right)_{, r}\right\|_{L_{2,-\mu}\left(\mathbb{R}^{3}\right)} \leq c\left\|\bar{f}_{\delta}\right\|_{L_{2,-\mu}\left(\mathbb{R}^{3}\right)} .
$$


By the Hardy inequality (see [1]) we have

$$
\begin{aligned}
& \int_{\mathbb{R}^{3}}\left|\bar{v}_{\delta}\right|^{2} r^{-2 \mu-4} r d r d z d \varphi \leq c \int_{\mathbb{R}^{3}}\left|\bar{v}_{\delta, r}\right|^{2} r^{-2 \mu-2} r d r d z d \varphi \\
& \leq c \int_{\mathbb{R}^{3}}\left|r \bar{v}_{\delta, r}\right|^{2} r^{-2 \mu-3} d r d z d \varphi \leq c \int_{\mathbb{R}^{3}}\left|\left(r \bar{v}_{\delta, r}\right)_{, r}\right|^{2} r^{-2 \mu-1} d r d z d \varphi
\end{aligned}
$$$$
=\int_{\mathbb{R}^{3}}\left|\frac{1}{r}\left(r \bar{v}_{\delta, r}\right)_{, r}\right|^{2} r^{-2 \mu} r d r d z d \varphi \leq c \int_{\mathbb{R}^{3}}\left|\bar{f}_{\delta}\right|^{2} r^{-2 \mu} r d r d z d \varphi=c\left\|\bar{f}_{\delta}\right\|_{L_{2,-\mu}\left(\mathbb{R}^{3}\right)}^{2} .
$$

To justify all inequalities in (2.8) we show the continuity of $\bar{v}_{\delta}$ at $r=\delta$. Integrating (2.6) with respect to $r$ from $r=\delta / 2$ to $r=\varrho, \varrho \in(\delta, 2 \delta)$, and with respect to $\varphi$ and $z$, we obtain

$$
\begin{array}{r}
\int_{\mathbb{R}} d z \int_{0}^{2 \pi} d \varphi \int_{\delta / 2}^{\varrho} \partial_{r}\left(r \bar{v}_{\delta, r}\right) d r+\int_{\mathbb{R}} d z \int_{0}^{2 \pi} d \varphi \int_{\delta / 2}^{\varrho} r d r\left(\frac{1}{r^{2}} \bar{v}_{\delta, \varphi \varphi}+\bar{v}_{\delta, z z}\right) \\
=\int_{\mathbb{R}} d z \int_{0}^{2 \pi} d \varphi \int_{\delta / 2}^{\varrho} \bar{h}_{\delta} r d r
\end{array}
$$

Since $\bar{v}_{\delta}$ is periodic with respect to $\varphi$ and vanishes for $|z|$ sufficiently large, the second term on the l.h.s. of (2.9) vanishes. Hence (2.9) takes the form

$$
\int_{\mathbb{R}} d z \int_{0}^{2 \pi} d \varphi \varrho \bar{v}_{\delta, r}(\varrho)-\int_{\mathbb{R}} d z \int_{0}^{2 \pi} d \varphi \frac{\delta}{2} \bar{v}_{\delta, r}\left(\frac{\delta}{2}\right)=\int_{\mathbb{R}} d z \int_{0}^{2 \pi} d \varphi \int_{\delta / 2}^{\varrho} \bar{h}_{\delta} r d r .
$$

But $\bar{v}_{\delta}$ vanishes for $r<\delta$ so the second integral on the 1.h.s. of $(2.10)$ disappears.

Assuming that $\tilde{h}_{\delta}$ has a compact support with respect to $z$ we obtain from (2.10) the inequality

$$
\left|\int_{\mathbb{R}} d z \int_{0}^{2 \pi} d \varphi \varrho \bar{v}_{\delta, r}(\varrho)\right| \leq c(\varrho-\delta)^{1 / 2}\left(\int_{\mathbb{R}} d z \int_{0}^{2 \pi} d \varphi \int_{\delta}^{\varrho}\left|\bar{h}_{\delta}\right|^{2} r d r\right)^{1 / 2} .
$$

Let us now assume that $\bar{h}_{\delta}$ is smooth. Then solutions to problem (2.6) are also smooth and estimate (2.3) holds. Let

$$
B_{\sigma}\left(\varphi_{0}, z_{0}\right)=\left\{(\varphi, z) \in[0,2 \pi] \times \mathbb{R}: \sqrt{\left(\varphi-\varphi_{0}\right)^{2}+\left(z-z_{0}\right)^{2}}<\sigma\right\} .
$$

Take $\varphi_{0}, z_{0}$ and $\sigma$ such that $\bar{v}_{\delta, r}(r)>0$ for $r \in(\delta, \varrho)$ and $(\varphi, z) \in B_{\sigma}\left(\varphi_{0}, z_{0}\right)$. Then (2.11) implies a contradiction.

Using that $H^{2}$ smooth functions are dense in $H^{2}$ we show that

$$
\lim _{\substack{r \rightarrow \delta \\ r>\delta}} \bar{v}_{\delta, r}(r)=0
$$


The last statement can also be proved in a different way. Assume that $f_{\delta} \in L_{2}\left(\mathbb{R}^{3}\right)$ is a smooth function vanishing for $r \leq \delta$. Then $\bar{h}_{\delta} \in L_{2}\left(\mathbb{R}^{3}\right)$ is also a smooth function. Hence by Lemma 2.1 there exists a smooth solution to problem (2.6) such that $\bar{v}_{\delta} \in H^{2}\left(\mathbb{R}^{3}\right)$. Integrating $(2.6)_{1}$ with respect to $r$ from $r=\delta / 2$ to $r=\delta$ we get

$$
r \bar{v}_{\delta, r}(\varrho)=-\int_{\delta}^{\varrho}\left(\frac{1}{r^{2}} \bar{v}_{\delta, \varphi \varphi}+\bar{v}_{\delta, z z}\right) r d r+\int_{\delta}^{\varrho} \bar{h}_{\delta} r d r
$$

so

$$
\begin{aligned}
\left|r \bar{v}_{\delta, r}(\varrho)\right| \leq & (\varrho-\delta)^{1 / 2}\left[\int_{\delta}^{\varrho} 2\left(\frac{1}{r^{4}} \bar{v}_{\delta, \varphi \varphi}^{2}+\bar{v}_{\delta, z z}^{2}\right) r^{2} d r\right]^{1 / 2} \\
& +(\varrho-\delta)^{1 / 2}\left[\int_{\delta}^{\varrho} \bar{h}_{\delta}^{2} r^{2} d r\right]^{1 / 2} .
\end{aligned}
$$

Hence for smooth solutions in $H^{2}\left(\mathbb{R}^{3}\right)$ we have

$$
\lim _{\substack{\varrho \rightarrow \delta \\ \varrho>\delta}} \bar{v}_{\delta, r}(\varrho)=0 .
$$

By the density argument we infer that (2.12) holds for solutions in $H^{2}\left(\mathbb{R}^{3}\right)$. Hence $\bar{v}_{\delta} \in H_{-\mu}^{2}\left(\mathbb{R}^{3}\right)$. Applying [2, 7] we obtain the estimate

$$
\left\|\bar{v}_{\delta}\right\|_{H_{-\mu}^{2}\left(\mathbb{R}^{3}\right)} \leq c\left\|\bar{f}_{\delta}\right\|_{L_{2,-\mu}\left(\mathbb{R}^{3}\right)},
$$

where $c$ does not depend on $\delta$.

Passing to the Cartesian coordinates and taking the limit $\delta \rightarrow 0$ we obtain

Lemma 2.2. Assume that $f \in L_{2,-\mu}\left(\mathbb{R}^{3}\right), \mu \in \mathbb{R}_{+} \backslash \mathbb{Z}$. Then there exists a solution to the problem

$$
\begin{array}{ll}
-\Delta u=f & \text { in } \mathbb{R}^{3}, \\
u \rightarrow 0 & \text { as } r \rightarrow \infty,|z| \rightarrow \infty,
\end{array}
$$

such that $u \in H_{-\mu}^{2}\left(\mathbb{R}^{3}\right)$ and the estimate

$$
\|u\|_{H_{-\mu}^{2}\left(\mathbb{R}^{3}\right)} \leq c\|f\|_{L_{2,-\mu}\left(\mathbb{R}^{3}\right)}
$$

holds.

Let us consider the case $l>0$. We have

Lemma 2.3. Assume that $f_{\delta} \in H^{l}\left(C_{R, \delta}\right)$. Then there exists a unique solution to problem (2.2) such that $u_{\delta} \in H^{l+2}\left(C_{R, \delta}\right)$ and

$$
\left\|u_{\delta}\right\|_{H^{l+2}\left(C_{R, \delta}\right)} \leq c\left\|f_{\delta}\right\|_{H^{l}\left(C_{R, \delta}\right)} .
$$


Finally, we prove a lemma similar to Lemma 2.2 for $f \in H_{-\mu}^{l}\left(\mathbb{R}^{3}\right)$. Let us consider problem (2.4). In view of Lemma $2.3, f_{\delta}^{\prime \prime} \in H^{l}\left(\mathbb{R}^{3} \backslash \bar{C}_{\delta}\right)$, so there exists a solution to problem (2.4) such that $u_{\delta}^{\prime} \in H^{l+2}\left(\mathbb{R}^{3} \backslash \bar{C}_{\delta}\right)$ and

$$
\left\|u_{\delta}^{\prime}\right\|_{H^{l+2}\left(\mathbb{R}^{3} \backslash \bar{C}_{\delta}\right)} \leq c\left\|f_{\delta}^{\prime \prime}\right\|_{H^{l}\left(\mathbb{R}^{3} \backslash \bar{C}_{\delta}\right)} .
$$

Let us recall the extensions denoted by $\bar{v}_{\delta}$ and $\bar{h}_{\delta}$. Assuming that $\bar{h}_{\delta} \in$ $H_{-\mu}^{l}\left(\mathbb{R}^{3}\right)$ and applying a recurrence argument based on the proof of Lemma 2.2 we have

$$
\begin{array}{r}
\left\|\frac{1}{r}\left(r \bar{v}_{\delta, r}\right)_{, r}\right\|_{H_{-\mu}^{l}\left(\mathbb{R}^{3}\right)}+\left\|\frac{1}{r^{2}} \bar{v}_{\delta, \varphi \varphi}\right\|_{H_{-\mu}^{l}\left(\mathbb{R}^{3}\right)}+\left\|\bar{v}_{\delta, z z}\right\|_{H_{-\mu}^{l}\left(\mathbb{R}^{3}\right)} \\
\leq c\left\|\bar{f}_{\delta}\right\|_{H_{-\mu}^{l}\left(\mathbb{R}^{3}\right)} .
\end{array}
$$

Hence by the Hardy inequality and considerations from the proof of Lemma 2.2 it follows that $\bar{v}_{\delta} \in H_{-\mu}^{l+2}\left(\mathbb{R}^{3}\right)$. Then [7] yields the estimate

$$
\left\|\bar{v}_{\delta}\right\|_{H_{-\mu}^{l+2}\left(\mathbb{R}^{3}\right)} \leq c\left\|\bar{f}_{\delta}\right\|_{H_{-\mu}^{l}\left(\mathbb{R}^{3}\right)},
$$

where $c$ does not depend on $\delta$. Passing to the limit $\delta \rightarrow 0$ we obtain

Lemma 2.4. Assume that $f \in H_{-\mu}^{l}\left(\mathbb{R}^{3}\right), \mu \in \mathbb{R}_{+} \backslash \mathbb{Z}, l \in \mathbb{N}_{0}$. Then there exists a solution to problem (2.14) such that $u \in H_{-\mu}^{l+2}\left(\mathbb{R}^{3}\right)$ and the estimate

$$
\|u\|_{H_{-\mu}^{l+2}\left(\mathbb{R}^{3}\right)} \leq c\|f\|_{H_{-\mu}^{l}\left(\mathbb{R}^{3}\right)}
$$

holds.

3. Existence in a bounded domain. The aim of this section is to prove Theorem 1.2. For this purpose we use the regularizer technique so we need a partition of unity. Let us define two collections of open subsets $\left\{\omega^{(k)}\right\}$ and $\left\{\Omega^{(k)}\right\}, k \in \bigcup_{i=1}^{4} \mathcal{M}_{i}$, such that $\bar{\omega}^{(k)} \subset \Omega^{(k)}, \bigcup_{k} \omega^{(k)}=\bigcup_{k} \Omega^{(k)}=\Omega$, $\Omega^{(k)} \cap S=\emptyset$ for $k \in \mathcal{M}_{1} \cup \mathcal{M}_{3}$ and $\Omega^{(k)} \cap S \neq \emptyset$ for $k \in \mathcal{M}_{2} \cup \mathcal{M}_{4}$. Here $\Omega^{(k)}, k \in \mathcal{M}_{1}$, is a neighbourhood of an interior point of $L \cap \Omega ; \Omega^{(k)}$, $k \in \mathcal{M}_{2}$, is a neighbourhood of a point where $L$ meets $S ; \Omega^{(k)}, k \in \mathcal{M}_{3}$, is a neighbourhood of an interior point of $\Omega$, located at a positive distance from $L ; \Omega^{(k)}, k \in \mathcal{M}_{4}$, is a neighbourhood of a point of $S$, located at a positive distance from $L$. We assume that at most $N_{0}$ of $\Omega^{(k)}$ 's have nonempty intersection, and $\sup _{k} \operatorname{diam} \Omega^{(k)} \leq 2 \lambda$ for some $\lambda>0$.

Let $\zeta^{(k)}(x)$ be a smooth function such that $0 \leq \zeta^{(k)}(x) \leq 1, \zeta^{(k)}(x)$ $=1$ for $x \in \omega^{(k)}, \operatorname{supp} \zeta^{(k)} \subset \Omega^{(k)}$ and $\left|D_{x}^{\nu} \zeta^{(k)}(x)\right| \leq c /|\lambda|^{\nu}$. Then $1 \leq$ $\sum_{k}\left(\zeta^{(k)}(x)\right)^{2} \leq N_{0}$. Introducing the function $\eta^{(k)}(x)=\zeta^{(k)}(x) / \sum_{l}\left(\zeta^{(l)}(x)\right)^{2}$ we have $\operatorname{supp} \eta^{(k)} \subset \Omega^{(k)}, \sum_{k} \eta^{(k)}(x) \zeta^{(k)}(x)=1,\left|D_{x}^{\nu} \eta^{(k)}\right| \leq c /|\lambda|^{\nu}$. By $\xi^{(k)}$ we denote a fixed interior point of $\omega^{(k)}$ and $\Omega^{(k)}$ for $k \in \mathcal{M}_{1} \cup \mathcal{M}_{3}$, and a point of $\overline{\omega^{(k)}} \cap S$ and $\overline{\Omega^{(k)}} \cap S$ for $k \in \mathcal{M}_{2} \cup \mathcal{M}_{4}$. 
Since we consider a problem invariant with respect to translations and rotations we can introduce a local coordinate system $y=\left(y_{1}, y_{2}, y_{3}\right)$ centred at $\xi^{(k)}$ such that for $k \in \mathcal{M}_{2} \cup \mathcal{M}_{4}$ the part $\tilde{S}^{(k)}=S \cap \overline{\Omega^{(k)}}$ of the boundary is described by $y_{3}=F\left(y_{1}, y_{2}\right)$. We assume that a point with coordinates $\left(y_{1}, y_{2}, y_{3}\right), y_{3}>F\left(y_{1}, y_{2}\right)$, belongs to $\Omega$. Then we introduce new coordinates by

$$
z_{i}=y_{i}, \quad i=1,2, \quad z_{3}=y_{3}-F\left(y_{1}, y_{2}\right) .
$$

We denote by $\Psi_{k}$ the transformation $\Omega^{(k)} \ni y \mapsto \Psi_{k}(y)=z \in \hat{\Omega}^{(k)}$, described by (3.1), such that $\omega^{(k)} \ni y \mapsto \Psi_{k}(y)=z \in \hat{\omega}^{(k)}$. We assume that the sets $\hat{\omega}^{(k)}, \hat{\Omega}^{(k)}$ are described in local coordinates at $\xi^{(k)}$ by the inequalities

$$
\begin{array}{ll}
\left|y_{i}\right|<\lambda, & i=1,2, \quad 0<y_{3}-F\left(y_{1}, y_{2}\right)<\lambda, \\
\left|y_{i}\right|<2 \lambda, & i=1,2, \quad 0<y_{3}-F\left(y_{1}, y_{2}\right)<2 \lambda,
\end{array}
$$

respectively.

Let $y=Y_{k}(x)$ be a transformation from the $x$ coordinates to local coordinates with origin at $\xi^{(k)}$ which is a composition of a translation and a rotation.

We denote $\Phi_{k}=\Psi_{k} \circ Y_{k}$. Then we set

$$
\hat{u}^{(k)}(z)=u\left(\Phi_{k}^{-1}(z)\right), \quad \tilde{u}^{(k)}(z)=\hat{u}^{(k)}(z) \hat{\zeta}^{(k)}(z) .
$$

First, we prove

Lemma 3.1. Assume that $f \in L_{2,-\mu}(\Omega), \mu \in(0,1)$. Then there exists a solution to problem (1.1) such that $u \in H_{-\mu}^{2}(\Omega)$ and

$$
\|u\|_{H_{-\mu}^{2}(\Omega)} \leq c\|f\|_{L_{2,-\mu}(\Omega)} .
$$

Proof. Since $f \in L_{2,-\mu}(\Omega), \mu>0$, we have $f \in L_{2}(\Omega)$ and

$$
\|f\|_{L_{2}(\Omega)} \leq c\|f\|_{L_{2,-}(\Omega)},
$$

because $\Omega$ is bounded. Then we have the existence of solutions to problem (1.1) such that $u \in H^{2}(\Omega)$ and

$$
\|u\|_{H^{2}(\Omega)} \leq c\|f\|_{L_{2}(\Omega)} .
$$

To prove the lemma, first we have to consider the problem (1.1) locally. Take $\xi^{(k)} \in L \cap \Omega, k \in \mathcal{M}_{1}$. Let us introduce a local Cartesian system $y=\left(y_{1}, y_{2}, y_{3}\right)$ with the origin at $\xi^{(k)}$ such that $L$ is the $y_{3}$ axis.

Let $R$ and $a$ be given positive numbers. Let $Q$ be a cylinder of the form

$$
Q=\left\{y \in \mathbb{R}^{3}:\left|y^{\prime}\right|<R,\left|y_{3}\right|<a\right\},
$$

where $y^{\prime}=\left(y_{1}, y_{2}\right)$.

Assume that $\Omega^{(k)} \subset Q, k \in \mathcal{M}_{1}$. Let $\zeta=\zeta^{(k)}(y), k \in \mathcal{M}_{1}$, be a smooth function from the partition of unity such that $\operatorname{supp} \zeta \subset Q$. Let $\tilde{u}=u \zeta$, 
$\tilde{f}=f \zeta$. Then problem (1.1) takes the form

$$
\begin{aligned}
& -\Delta \tilde{u}=\tilde{f}-2 \nabla \zeta \nabla u-\Delta \zeta u \equiv \tilde{f}_{0}, \\
& \left.\tilde{u}\right|_{\partial Q}=0,
\end{aligned}
$$

where we consider the case $Q \cap S=\emptyset$.

To apply Lemma 2.2 we have to know that $\tilde{f}_{0} \in L_{2,-\mu}\left(\mathbb{R}^{3}\right)$. Since $\mu \in$ $(0,1)$ and in view of $(3.6)$ we know that $\tilde{u} \in H^{2}\left(\mathbb{R}^{3}\right)$, the Hardy inequality implies

$$
\nabla u \in L_{2,-\mu}\left(\mathbb{R}^{3} \cap Q\right), \quad u \in L_{2,-\mu}\left(\mathbb{R}^{3} \cap Q\right) .
$$

Then Lemma 2.2 implies the existence of solutions to problem (3.7) such that $\tilde{u} \in H_{-\mu}^{2}\left(\mathbb{R}^{3}\right)$ and

$$
\|\tilde{u}\|_{H_{-\mu}^{2}\left(\mathbb{R}^{3}\right)} \leq c\|f\|_{L_{2,-\mu}(\Omega)}
$$

because

$$
\|\nabla u\|_{L_{2,-\mu}\left(\mathbb{R}^{3} \cap Q\right)}+\|u\|_{L_{2,-\mu}\left(\mathbb{R}^{3} \cap Q\right)} \leq c\|f\|_{L_{2}(\Omega)} .
$$

Let $\xi^{(k)}, k \in \mathcal{M}_{2}$, be a point where $L$ meets $S$. Let us introduce a local system of coordinates $y=\left(y_{1}, y_{2}, y_{3}\right)$ with the origin at $\xi^{(k)}$. Then in the subdomain $\Omega^{(k)}, k \in \mathcal{M}_{2}$, the part of the boundary $\tilde{S}^{(k)}=S \cap \overline{\Omega^{(k)}}$ is described by

$$
y_{3}=F\left(y_{1}, y_{2}\right),
$$

where $F(0,0)=0$. Let $\hat{\nabla}_{i}=\frac{\partial z_{k}}{\partial x_{i}} \frac{\partial}{\partial z_{k}}$, where the summation convention over the repeated indices is assumed, and $z=\Phi_{k}(x)$, where $k \in \mathcal{M}_{2}$. Then problem (1.1) in the local system of coordinates takes the form

$$
\begin{aligned}
& -\hat{\nabla}_{i}^{2} \hat{u}^{(k)}=\hat{f}^{(k)}, \quad z_{3}>0, \\
& \left.\hat{u}^{(k)}\right|_{z_{3}=0}=0,
\end{aligned}
$$

for $k \in \mathcal{M}_{2}$. Let us extend problem (3.9) to $z_{3}<0$ by reflection. We denote the extended functions by $\hat{u}^{(k) \prime}, \zeta^{\prime}$. Let $\hat{\zeta}=\hat{\zeta}^{(k)}, k \in \mathcal{M}_{2}$, be a function from the partition of unity, $\hat{\zeta}^{\prime}$ be the extension of $\hat{\zeta}$ and let $\operatorname{supp} \hat{\zeta}^{\prime} \subset Q^{\prime}$, where

$$
Q^{\prime}=\left\{z \in \mathbb{R}^{3}:\left|z^{\prime}\right|<R,\left|z_{3}\right|<a\right\}
$$

Set $\tilde{u}=\hat{u}^{(k)^{\prime}} \hat{\zeta}^{\prime}, \tilde{f}=\hat{f}^{(k)^{\prime}} \hat{\zeta}^{\prime}$. Then (3.9) assumes the form

$$
\begin{aligned}
& -\hat{\nabla}_{i}^{2} \tilde{u}=\tilde{f}-2 \hat{\nabla} \hat{u}^{\prime} \cdot \nabla \hat{\zeta}^{\prime}-\hat{\Delta} \hat{\zeta}^{\prime} \hat{u}^{\prime} \equiv \tilde{f}_{0}^{\prime}, \\
& \left.\tilde{u}\right|_{\partial Q^{\prime}}=0,
\end{aligned}
$$

where $\hat{u}^{\prime}=\hat{u}^{(k) \prime}, \hat{\Delta}=\hat{\nabla}_{i}^{2}$. In view of (3.6) we have $\tilde{f}_{0}^{\prime} \in L_{2,-\mu}\left(Q^{\prime}\right), \mu \in(0,1)$. Then Lemma 2.2 implies that $\tilde{u} \in H_{-\mu}^{2}\left(\mathbb{R}^{3}\right)$.

Moreover, from (3.6) it follows that $\tilde{u} \in H_{-\mu}^{2}\left(\mathbb{R}^{3}\right)$ in neighbourhoods located at a positive distance from $L$. 
To introduce the regularizer (see [4]) we define all local problems in a uniform way. Let $k \in \mathcal{M}_{1}$. Then problem (3.7) is expressed as

$$
-\nabla_{x}^{2} \tilde{u}^{(k)}=\tilde{f}_{*}^{(k)} \text { in } \mathbb{R}^{3} .
$$

For $k \in \mathcal{M}_{2},(3.10)$ takes the form

$$
-\nabla_{z}^{2} \tilde{u}^{(k)}=\tilde{f}_{*}^{(k)} \text { in } \mathbb{R}^{3} .
$$

For $k \in \mathcal{M}_{3}$, we consider the problem

$$
-\nabla_{x}^{2} u^{(k)}=\tilde{f}_{*}^{(k)} \text { in } \mathbb{R}^{3} .
$$

Finally, for $k \in \mathcal{M}_{4}$ we have

$$
\begin{array}{rlrl}
-\nabla_{z}^{2} u^{(k)} & =\tilde{f}_{*}^{(k)} & & \text { in } \mathbb{R}_{+}^{3}, \\
\left.u^{(k)}\right|_{z_{3}=0}=0 & & \text { on } \mathbb{R}^{2} .
\end{array}
$$

By Lemma 2.2 we have the existence of solutions to problems (3.11) and (3.12). Since neighbourhoods $\Omega^{(k)}, k \in \mathcal{M}_{3} \cup \mathcal{M}_{4}$, are located at a positive distance from $L$ we have the existence in $H^{l+2}$ so also in $H_{-\mu}^{l+2}$ of solutions to problems (3.13) and (3.14).

Let $R^{(k)}$ be the operator which solves the $k$ th problem. Then we define the operator

$$
R f=\sum_{k \in \mathcal{M}} \eta^{(k)}(x) u^{(k)}(x)
$$

where

$$
u^{(k)}(x)= \begin{cases}R^{(k)} \zeta^{(k)} f & \text { for } k \in \mathcal{M}_{1} \cup \mathcal{M}_{3}, \\ \Phi_{k}^{-1} R^{(k)}\left(\Phi_{k} \zeta^{(k)} f\right) & \text { for } k \in \mathcal{M}_{2} \cup \mathcal{M}_{4} .\end{cases}
$$

Let us introduce the spaces $H=H(\Omega)=L_{2,-\mu}, V=V(\Omega)=H_{-\mu}^{2}, \mu \in$ $(0,1)$, endowed with the norms

$$
\|f\|_{H}=\sum_{k \in \mathcal{M}}\left\|f^{(k)}\right\|_{L_{2,-\mu}\left(\mathbb{R}^{(k)}\right)}, \quad\|u\|_{V}=\sum_{k \in \mathcal{M}}\left\|u^{(k)}\right\|_{H_{-\mu}^{2}\left(\mathbb{R}^{(k)}\right)},
$$

where

$$
\mathbb{R}^{(k)}= \begin{cases}\mathbb{R}^{3} & \text { for } k \in \mathcal{M}_{1} \cup \mathcal{M}_{2} \cup \mathcal{M}_{3}, \\ \mathbb{R}_{+} & \text {for } k \in \mathcal{M}_{4} .\end{cases}
$$

Since solvability of problems (3.11)-(3.14) is known we deduce $R: H \rightarrow V$ is a bounded operator. Let $\mathcal{Z}$ be the operator of problem (1.1). It will be shown that for $f \in H$ we have

$$
\mathcal{Z} R f=\left(I+T_{1}+T_{2}\right) f, \quad T=T_{1}+T_{2},
$$

where $I$ is the identity operator, $T_{1}: H \rightarrow H$ with the norm $\left\|T_{1}\right\|<1$, and $T_{2}$ is a completely continuous operator. Moreover, for $v \in V$ we also have

$$
R \mathcal{Z} v=\left(I+W_{1}+W_{2}\right) v, \quad W=W_{1}+W_{2},
$$


where $W_{1}: V \rightarrow V$ with $\left\|W_{1}\right\|<1$ and $W_{2}$ is a completely continuous operator.

Since the operators $\left(I+T_{1}\right)^{-1}$ and $\left(I+W_{1}\right)^{-1}$ are bounded, we can express (3.15) and (3.16) in the form

$$
\mathcal{Z} R^{\prime} f^{\prime}=\left(I+T_{2}^{\prime}\right) f^{\prime}
$$

and

$$
R^{\prime \prime} \mathcal{Z} v=\left(I+W_{2}^{\prime}\right) v
$$

where

$$
\begin{gathered}
R^{\prime}=R\left(I+T_{1}\right)^{-1}, \quad f^{\prime}=\left(I+T_{1}\right) f, \quad T_{2}^{\prime}=T_{2}\left(I+T_{1}\right)^{-1}, \\
R^{\prime \prime}=\left(I+W_{1}\right)^{-1} R, \quad W_{2}^{\prime}=\left(I+W_{1}\right)^{-1} W_{2} .
\end{gathered}
$$

Moreover, the operators $T_{2}^{\prime}$ and $W_{2}^{\prime}$ are also completely continuous.

From (3.16) we obtain

$$
\|v\|_{H_{-\mu}^{2}(\Omega)} \leq c\left(\|R \mathcal{Z} v\|_{H_{-\mu}^{2}(\Omega)}+\left\|W_{1} v\right\|_{H_{-\mu}^{2}(\Omega)}+\left\|W_{2} v\right\|_{H_{-\mu}^{2}(\Omega)}\right) .
$$

From the properties of $R$ and the fact that the norm of $W_{1}$ is less than one we obtain from (3.19) the inequality

$$
\|v\|_{H_{-\mu}^{2}(\Omega)} \leq c\left(\|\mathcal{Z} v\|_{L_{2,-\mu}(\Omega)}+\left\|W_{2} v\right\|_{H_{-\mu}^{2}(\Omega)}\right) .
$$

Since $W_{2}$ is completely continuous with

$$
\left\|W_{2} v\right\|_{H_{-\mu}^{2}(\Omega)} \leq \varepsilon\|v\|_{H_{-\mu}^{2}(\Omega)}+c(1 / \varepsilon)\|v\|_{L_{2}(\Omega)},
$$

where $\varepsilon \in(0,1)$ and $c(1 / \varepsilon) \sim \varepsilon^{-a}, a>0$, we have

$$
\|v\|_{H_{-\mu}^{2}(\Omega)} \leq c\left(\|\mathcal{Z} v\|_{L_{2,-\mu}(\Omega)}+\|v\|_{L_{2}(\Omega)}\right) .
$$

Having the existence of weak solutions to problem $(1.1)$ in $H^{1}(\Omega)$ we obtain from (3.20) the estimate

$$
\|v\|_{H_{-\mu}^{2}(\Omega)} \leq c\|\mathcal{Z} v\|_{L_{2,-\mu}(\Omega)} .
$$

Hence there exists an inverse operator $\mathcal{Z}^{-1}$ for problem (1.1) so we have the existence of solution to problem (1.1) in $H_{-\mu}^{2}(\Omega), \mu \in(0,1)$. We can prove the existence in a more explicit form. Let us express (3.16) in the form

$$
v-W_{1} v=R \mathcal{Z} v+W_{2} v .
$$

Having the existence in $H^{1}(\Omega)$ the r.h.s. of (3.22) belongs to $H_{-\mu}^{2}(\Omega)$. Since $W_{1}$ has norm less than one we prove the existence of solutions to (3.22) in $H_{-\mu}^{2}(\Omega)$ by a fixed point argument.

We have to mention that the argument of the operator $W_{2}$ vanishes in a neighbourhood of $L$. Thus Lemma 3.1 will be proved. 
Now we derive the forms of the operators $T_{1}, T_{2}, W_{1}, W_{2}$ and show the above statements. Following [4] we have

$$
\mathcal{Z} R f=\sum_{k \in \mathcal{M}}\left(\mathcal{Z} \eta^{(k)} u^{(k)}-\eta^{(k)} \mathcal{Z} u^{(k)}\right)+\sum_{k \in \mathcal{M}} \eta^{(k)} \mathcal{Z} u^{(k)} .
$$

For $k \in \mathcal{M}_{1} \cup \mathcal{M}_{3}$ we have

$$
\mathcal{Z} u^{(k)}=\mathcal{Z} R^{(k)} f^{(k)}=f^{(k)}=\zeta^{(k)} f .
$$

For $k \in \mathcal{M}_{2} \cup \mathcal{M}_{4}$,

$$
\begin{aligned}
\mathcal{Z}\left(\partial_{x}\right) u^{(k)}= & \mathcal{Z}\left(\partial_{x}\right) \Phi_{k}^{-1} R^{(k)}\left(\Phi_{k} \zeta^{(k)} f\right) \\
= & \Phi_{k}^{-1} \mathcal{Z}\left(\partial_{z}-\nabla F \cdot \partial_{z_{3}}\right) R^{(k)}\left(\Phi_{k} \zeta^{(k)} f\right) \\
= & \Phi_{k}^{-1}\left[\mathcal{Z}\left(\partial_{z}-\nabla F \partial_{z_{3}}\right)-\mathcal{Z}\left(\partial_{z}\right)\right] R^{(k)}\left(\Phi_{k} \zeta^{(k)} f\right) \\
& +\Phi_{k}^{-1} \mathcal{Z}\left(\partial_{z}\right) R^{(k)}\left(\Phi_{k} \zeta^{(k)} f\right),
\end{aligned}
$$

where for $k \in \mathcal{M}_{4}$ we should write $\left(\zeta^{(k)} f, 0\right)$ instead of $\zeta^{(k)} f$. In view of the definition of $R^{(k)}$ the last expression in (3.23) equals $\zeta^{(k)} f$. Therefore, the operator $T$ takes the form

$$
\begin{aligned}
T f= & \sum_{k \in \mathcal{M}}\left(\mathcal{Z} \eta^{(k)} u^{(k)}-\eta^{(k)} \mathcal{Z} u^{(k)}\right) \\
& +\sum_{k \in \mathcal{M}_{2} \cup \mathcal{M}_{4}} \eta^{(k)} \Phi_{k}^{-1}\left[\mathcal{Z}\left(\partial_{z}-\nabla F \partial_{z_{3}}\right)-\mathcal{Z}\left(\partial_{z}\right)\right] R^{(k)}\left(\Phi_{k} \zeta^{(k)} f\right) \\
\equiv & T_{2} f+T_{1} f .
\end{aligned}
$$

Now we construct the operator $W$. We examine the expression $R \mathcal{Z} u$.

For $k \in \mathcal{M}_{1} \cup \mathcal{M}_{3}$,

$$
R^{(k)} \zeta^{(k)} \mathcal{Z} u=R^{(k)}\left(\zeta^{(k)} \mathcal{Z} u-\mathcal{Z} \zeta^{(k)} u\right)+R^{(k)} \mathcal{Z} \zeta^{(k)} u,
$$

where

$$
R^{(k)} \mathcal{Z} \zeta^{(k)} u=\zeta^{(k)} u
$$

For $k \in \mathcal{M}_{2} \cup \mathcal{M}_{4}$ we have

$$
R^{(k)} \Phi_{k} \zeta^{(k)} \mathcal{Z} u=R^{(k)} \Phi_{k}\left(\zeta^{(k)} \mathcal{Z} u-\mathcal{Z} \zeta^{(k)} u\right)+R^{(k)} \Phi_{k} \mathcal{Z} \zeta^{(k)} u
$$

where the last expression equals

$$
R^{(k)}\left[\mathcal{Z}\left(\partial_{z}-\nabla F \partial_{z_{3}}\right)-\mathcal{Z}\left(\partial_{z}\right)\right] \Phi_{k} \zeta^{(k)} u+R^{(k)} \mathcal{Z}\left(\partial_{z}\right) \Phi_{k} \zeta^{(k)} u
$$

and by the definition of $R^{(k)}$ the last term equals $\Phi_{k} \zeta^{(k)} u$. Finally, the operator $W$ assumes the form 


$$
\begin{aligned}
W u= & \left\{\sum_{k \in \mathcal{M}_{1} \cup \mathcal{M}_{3}} \eta^{(k)} R^{(k)}\left(\zeta^{(k)} \mathcal{Z}-\mathcal{Z} \zeta^{(k)}\right) u\right. \\
& \left.+\sum_{k \in \mathcal{M}_{2} \cup \mathcal{M}_{4}} \eta^{(k)} \Phi_{k}^{-1} R^{(k)}\left[\Phi_{k}\left(\zeta^{(k)} \mathcal{Z}-\mathcal{Z} \zeta^{(k)}\right) u\right]\right\} \\
& +\sum_{k \in \mathcal{M}_{2} \cup \mathcal{M}_{4}} \eta^{(k)} \Phi_{k}^{-1} R^{(k)}\left[\mathcal{Z}\left(\partial_{z}-\nabla F \partial_{z_{3}}\right)-\mathcal{Z}\left(\partial_{z}\right)\right] \Phi_{k} \zeta^{(k)} u \\
\equiv & W_{2} u+W_{1} u .
\end{aligned}
$$

From the form of $T$ we have

$$
\begin{aligned}
T_{1} f= & \sum_{k \in \mathcal{M}_{2} \cup \mathcal{M}_{4}} \eta^{(k)} \Phi_{k}^{-1}\left[-\nabla_{i} F \nabla_{i} F \partial_{z_{3}}^{2}+\nabla_{i} F \partial_{z_{i}} \partial_{z_{3}}\right. \\
& \left.-\nabla F_{i} \partial_{z_{3}} \partial_{z_{i}}-\nabla F_{i} \partial_{z_{3}}\left(\nabla F_{i}\right) \partial_{z_{3}}\right] R^{(k)}\left(\Phi_{k} \zeta^{(k)} f\right)
\end{aligned}
$$

and

$$
\begin{aligned}
T_{2} f= & \sum_{k \in \mathcal{M}}\left(\mathcal{Z} \eta^{(k)} u^{(k)}-\eta^{(k)} \mathcal{Z} u^{(k)}\right) \\
& -\sum_{k \in \mathcal{M}_{2} \cup \mathcal{M}_{4}} \eta^{(k)} \Phi_{k}^{-1}\left[\partial_{z_{i}} \nabla F_{i} \partial_{z_{3}}\right] R^{(k)}\left(\Phi_{k} \zeta^{(k)} f\right) .
\end{aligned}
$$

Since $|\partial F / \partial z| \leq c \lambda$ we have $\left\|T_{1} f\right\|_{L_{2,-\mu}} \leq c \lambda\|f\|_{L_{2,-\mu}}$, so for $\lambda$ sufficiently small we have $\left\|T_{1}\right\|_{H \rightarrow H}<1$.

Since the operator $T_{2}$ involves at most the first derivatives of $u^{(k)}$, the compact imbedding $H_{-\mu}^{2} \subset H_{-\mu}^{1}$ implies that $T_{2}$ is completely continuous. Examining $W$ we have

$$
\begin{aligned}
W_{1} u= & \sum_{k \in \mathcal{M}_{2} \cup \mathcal{M}_{4}} \eta^{(k)} \Phi_{k}^{-1} R^{(k)}\left[-\nabla_{i} F \nabla_{i} F \partial_{z_{3}}^{2}-2 \nabla_{i} F \partial_{z_{i} z_{3}}\right. \\
& \left.-\nabla F_{i} \partial_{z_{3}}\left(\nabla F_{i}\right) \partial_{z_{3}}\right] \Phi_{k} \zeta^{(k)} u
\end{aligned}
$$

and

$$
\begin{aligned}
W_{2} u= & \sum_{k \in \mathcal{M}_{1} \cup \mathcal{M}_{3}} \eta^{(k)} R^{(k)}\left(\zeta^{(k)} \mathcal{Z}-\mathcal{Z} \zeta^{(k)}\right) u \\
& +\sum_{k \in \mathcal{M}_{2} \cup \mathcal{M}_{4}} \eta^{(k)} \Phi_{k}^{-1} R^{(k)}\left[\Phi_{k}\left(\zeta^{(k)} \mathcal{Z}-\mathcal{Z} \zeta^{(k)}\right) u\right] \\
& -\sum_{k \in \mathcal{M}_{2} \cup \mathcal{M}_{4}} \eta^{(k)} \Phi_{k}^{-1} R^{(k)}\left(\partial_{z_{i}} \nabla F_{i} \partial_{z_{3}}\right) \Phi_{k} \zeta^{(k)} u .
\end{aligned}
$$

By the same arguments as above we have $\left\|W_{1} u\right\|_{V \rightarrow V}<1$ and $W_{2}$ is completely continuous.

Using estimates for solutions to problems (3.11)-(3.14) and the global estimate (3.6) we show estimates (3.21) and (3.22).

Hence for $f \in H(\Omega)$ and $v \in V(\Omega)$ there exists a solution to problem (1.1) in $V(\Omega)$. 
We have to stress that all considerations leading to (3.15) and (3.16) are done for $f \in H(\Omega)$ and $v \in V(\Omega)$. Therefore, choosing the spaces $H(\Omega)$ and $V(\Omega)$ we were able to prove the existence in $V(\Omega)$. Hence, if either $\left\|T_{1}\right\|>1$ or $\left\|W_{1}\right\|>1$ we could not have proved the existence in $V(\Omega)$. This concludes the proof.

Lemma 3.2. Assume that $f \in L_{2,-\mu}(\Omega), \mu \in(1,2), S \in C^{2}$. Then there exists a solution to problem (1.1) such that $u \in H_{-\mu}^{2}(\Omega)$ and

$$
\|u\|_{H_{-\mu}^{2}(\Omega)} \leq c\|f\|_{L_{2,-\mu}(\Omega)}, \quad \mu \in(1,2) .
$$

Proof. Since $f \in L_{2,-\mu}(\Omega), \mu \in(1,2)$ and $\Omega$ is bounded we have $f \in$ $L_{2,-\mu}(\Omega), \mu \in(0,1)$. Hence the assumption of Lemma 3.1 holds. Then by the Hardy inequality we get $\tilde{f}_{*}^{(k)} \in L_{2,-\mu}\left(\mathbb{R}^{3}\right)$ for $\mu \in(1,2), k \in \mathcal{M}_{1} \cup \mathcal{M}_{2}$. Then repeating the considerations from the proof of Lemma 3.1 and assuming that $H(\Omega)=L_{2,-\mu}, V(\Omega)=H_{-\mu}^{2}, \mu \in(1,2)$, we conclude the proof.

Continuing the considerations we obtain

Lemma 3.3. Assume that $f \in L_{2,-\mu}(\Omega), \mu \in(k, k+1), k \in \mathbb{N}_{0}, S \in C^{2}$. Then there exists a solution to problem (1.1) such that $u \in H_{-\mu}^{2}(\Omega)$ and

$$
\|u\|_{H_{-\mu}^{2}(\Omega)} \leq c\|f\|_{L_{2,-\mu}(\Omega)}, \quad \mu \in(k, k+1) .
$$

Finally, we prove

Lemma 3.4. Assume that $f \in H_{-\mu}^{l}(\Omega), \mu \in \mathbb{R}_{+} \backslash \mathbb{Z}, l \in \mathbb{N}_{0}, S \in C^{l+2}$. Then there exists a solution to problem (1.1) such that $u \in H_{-\mu}^{l+2}(\Omega)$ and

$$
\|u\|_{H_{-\mu}^{l+2}(\Omega)} \leq c\|f\|_{H_{-\mu}^{l}(\Omega)} .
$$

Proof. We prove the lemma recurrently. Take $l=1$. From Lemma 3.3 we find that the r.h.s. of (3.11) and (3.12) belong to $H_{-\mu}^{1}$. Then we apply the regularizer technique for $H=H_{-\mu}^{1}$ and $V=H_{-\mu}^{3}$. Thus we prove the existence of $u$ in $H_{-\mu}^{3}$ and (3.26) for $l=1$. Having (3.26) for $l=1$ we infer that the r.h.s. of (3.11) and (3.12) belong to $H_{-\mu}^{2}$, so applying the regularizer technique for $H=H_{-\mu}^{2}$ and $V=H_{-\mu}^{4}$ we prove the assertion and (3.26) for $l=2$.

Continuing the considerations we conclude the proof.

Acknowledgments. This research was partially supported by MNiSW grant no 1 PO 3A 02130.

\section{References}

[1] V. A. Kondrat'ev, Boundary value problems for elliptic equations in domains with conical and angular points, Trudy Moskov. Mat. Obshch. 16 (1967), 209-292 (in Russian). 
[2] A. Kubica and W. M. Zajączkowski, A priori estimates in weighted spaces for solutions of the Poisson and heat equations, Appl. Math. (Warsaw) 34 (2007), 431-444.

[3] V. P. Mikhaĭlov, Partial Differential Equations, Nauka, Moscow 1976 (in Russian).

[4] V. A. Solonnikov, On general boundary value problems for Douglis-Nirenberg elliptic systems, Trudy Mat. Inst. Steklov. 92 (1966), 233-297 (in Russian).

[5] V. A. Solonnikov and W. M. Zajaczkowski, On the Neumann problem for elliptic equations of second order in domains with edges on the boundary, Zap. Nauchn. Sem. LOMI 127 (1983), 7-48 (in Russian).

[6] E. Zadrzyńska and W. M. Zajączkowski, Global regular solutions with large swirl to the Navier-Stokes equations in a cylinder, J. Math. Fluid Mech. 11 (2009), 126-169.

[7] W. M. Zajączkowski, Existence of solutions to the (rot, div)-system in $L_{2}$-weighted spaces, Appl. Math. (Warsaw) 36 (2009), 83-106.

[8] -, Solvability of the heat equation in weighted Sobolev spaces, ibid. to appear.

[9] - Global axially symmetric solutions with large swirl to the Navier-Stokes equations, Topol. Methods Nonlinear Anal. 29 (2007), 295-331.

[10] - Global regular solutions to the Navier-Stokes equations in a cylinder, in: Banach Center Publ. 74, Inst. Math., Polish Acad. Sci., 2006, 235-255.

[11] - Global special regular solution to the Navier-Stokes equations in axially symmetric domains under boundary slip conditions, Dissertationes Math. 432 (2005).

[12] - Existence and regularity of solutions of some elliptic system in domains with edges, ibid. 274 (1988).

Wojciech M. Zajączkowski

Institute of Mathematics

Polish Academy of Sciences

Śniadeckich 8

00-956 Warszawa, Poland

E-mail: wz@impan.pl

and

Institute of Mathematics and Cryptology

Cybernetics Faculty

Military University of Technology

Kaliskiego 2

00-908 Warszawa, Poland

Received on 9.7.2009;

revised version on 24.8.2009

(2011) 
